\title{
miR-199a-5p induces cell invasion by suppressing E-cadherin expression in cutaneous squamous cell carcinoma
}

\author{
SHAOHUA WANG ${ }^{1}, \mathrm{KE} \mathrm{CAO}^{2}$, QUANYONG HE ${ }^{1}$, ZHAOQI YIN ${ }^{1}$ and JIANDA ZHOU ${ }^{1}$ \\ Departments of ${ }^{1}$ Burns and Plastic Surgery and ${ }^{2}$ Oncology, The Third Xiangya Hospital, \\ Central South University, Changsha, Hunan 410013, P.R. China
}

Received December 17, 2014; Accepted February 11, 2016

DOI: $10.3892 / \mathrm{ol} .2016 .4602$

\begin{abstract}
A growing quantity of evidence exists to suggest that microRNAs are significant regulators of multiple cellular processes. When expressed aberrantly in different types of cancer, including cutaneous squamous cell carcinoma (cSCC), they play key roles in tumorigenesis and progression. The aberrant expression of miR-199a-5p has been observed to contribute to carcinogenesis in various types of cancer. However, the role of miR-199a-5p in the progression of cSCC metastasis remains largely unknown. In this study, we determined that miR-199a-5p was the upstream regulator of CDH1 (E-cadherin) and that it could suppress the expression of E-cadherin in cSCC cells. In addition, miR-199a-5p mimics significantly induced cell invasion and the activity of matrix metalloproteinase (MMP)2 and MMP9 in cSCC cells. In conclusion, these results are likely to aid in elucidating the molecular mechanisms of cSCC progression. In addition, the findings provide a new theoretical basis to further investigate miR-199a-5p as a potential biomarker and a promising approach in cSCC treatment.
\end{abstract}

\section{Introduction}

Cutaneous squamous cell carcinoma (cSCC) is one of the most common types of skin cancer, and is responsible for $20 \%$ of skin cancer-associated mortalities yearly $(1,2)$. Although the risk factors of cSCC are well characterized, the molecular pathogenesis of this particular tumor type is still not well understood. As increasing numbers of patients succumb to cSCC, it is an urgent requirement to clarify the molecular mechanisms of this cancer and to develop novel and more effective treatment strategies against the malignancy.

Correspondence to: Mr. Jianda Zhou, Department of Burns and Plastic Surgery, The Third Xiangya Hospital, Central South University, 138 Tongzipo Road, Changsha, Hunan 410013, P.R. China

E-mail: jiandazhou2014@163.com

Key words: miR-199a-5p, cutaneous squamous cell carcinoma, E-cadherin, MMP2, MMP9
microRNA (miRNA), a class of naturally occurring, 17-25 nucleotide small noncoding small RNA, regulates the expression of genes through binding to the 3' untranslated regions of target mRNAs. miRNAs have emerged as key factors involved in several biological processes, including development, differentiation, cell proliferation and tumorigenesis $(3,4)$. Several studies have demonstrated that alterations in miRNA genes lead to tumor formation, and several miRNAs that regulate either tumor suppression or tumor formation have been identified (5-7). In previous studies, a number of dysregulated miRNAs were observed in cSCC $(8,9)$. Zhou et al demonstrated that miR-365 was overexpressed in cells and clinical specimens of cSCC (10). miR-31 is overexpressed in cSCC and regulates cancer-associated phenotypes of cSCC (11). The decreased expression of the miR-193b/365a cluster during tumor progression suggests a tumor suppressor role of cSCC (12).

Previous studies have demonstrated that miR-199a-5p expression is deregulated in various cancer types $(7,13)$. For example, downregulated miR-199a-5p expression was observed in small-cell carcinoma and hepatocellular carcinoma $(14,15)$. Decreased expression of miR-199a-5p contributes to increased cell invasion by functional deregulation of discoidin domain receptor 1 activity (15). Forced expression of miR-199a-5p promoted cisplatin-induced inhibition of cell proliferation by targeting autophagy-associated gene 7 in hepatocellular carcinoma (16). In addition, the increased expression of miR-199a-5p was observed in gastric and colorectal cancer $(17,18)$. However, the roles and underlying molecular mechanisms of miR-199a-5p in cSCC are not completely understood.

This study revealed that miR-199a-5p is an upstream regulator of $\mathrm{CDH} 1$ (E-cadherin) which suppresses the expression of E-cadherin in cSCC cells. To further analyze the oncogenic role of miR-199a-5p in cSCC progression, we analyzed the effect of miR-199a-5p on cell invasion and the activity of matrix metalloproteinase (MMP)2 and MMP9 in cSCC cells.

\section{Materials and methods}

Cell culture and treatment. A-431 SCC cells were obtained from the American Type Culture Collection (ATCC; Manassas, VA, USA) and cultivated in RPMI-1640 medium with a final concentration of $10 \%$ fetal bovine serum. Cells were cultured in conditions of $95 \%$ air and $5 \%$ carbon dioxide at $37^{\circ} \mathrm{C}$. 
A

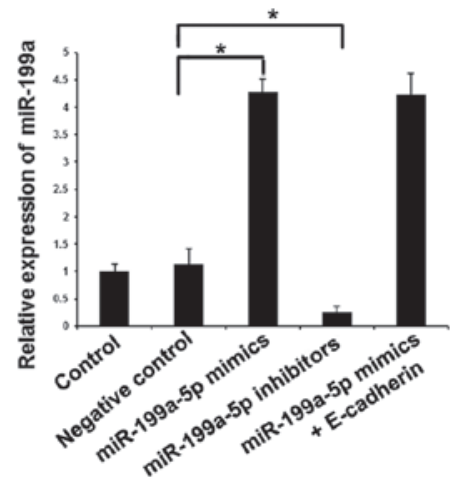

B

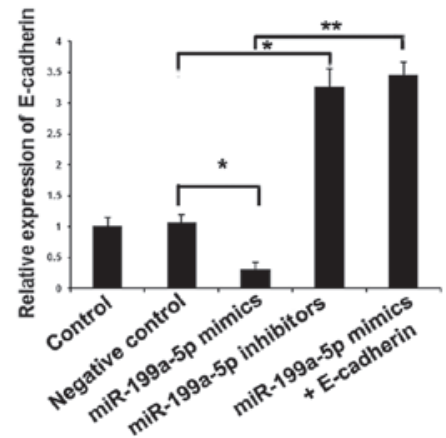

C
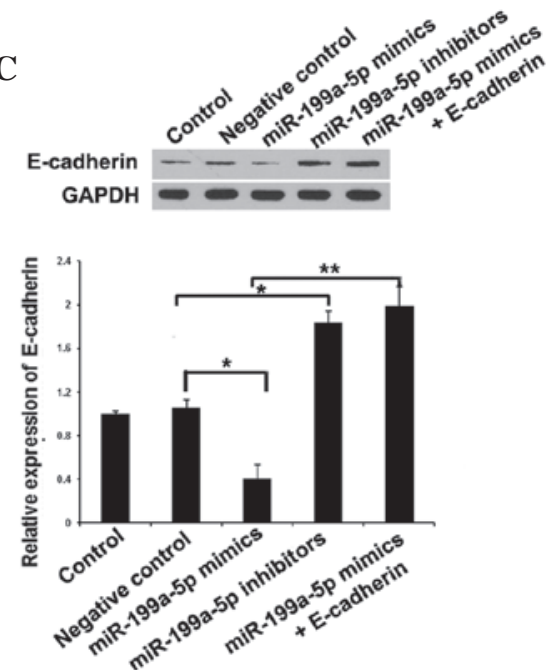

Figure 1. miR-199a-5p regulates the expression levels of E-cadherin in A-431 cells. (A) Reverse transcription-quantitative polymerase chain reaction (RT-qPCR) revealed the expression of miR-199a-5p. (B) qRT-PCR revealed the expression of E-cadherin. (C) Western blot analysis revealed the expression of E-cadherin protein. A-431 cells were transfected with miR-199a-5p mimics and inhibitors, or cotransfected with miR-199a-5p mimics and E-cadherin. Error bars represent the means \pm standard error. ${ }^{*} \mathrm{P}<0.01$ vs. negative control group; ${ }^{* *} \mathrm{P}<0.01$ vs. miR-199a-5p mimics group.

Ectopic expression of miR-199a-5p was achieved in the cells by transfection with miR-199a-5p mimics or inhibitors using Lipofectamine 2000 (Invitrogen Life Technologies, Carlsbad, CA, USA). Cells were plated in 24-well plates and transfected for $24 \mathrm{~h}$ or $48 \mathrm{~h}$. Total RNA or protein was extracted from the indicated cells for analysis.

Quantitative polymerase chain reaction ( $q P C R$ ) assay. Total RNA was extracted from the indicated cells according to the manufacturer's instructions using the Qiagen RNeasy kit (Qiagen Nordic, Solna, Sweden). The expression of CD44 and Ezrin mRNA was assessed by SYBR-Green qPCR assay (Bio-Rad Laboratories, Inc., Hercules, CA, USA). $\beta$-actin was used as an endogenous control. The specific primers were as follows: E-cadherin: F, cgacccaacccaagaatcta; R, aggctgtgcettcctacaga; and $\beta$-actin: $\mathrm{F}$, cattaaggagaagctgtgct; $\mathrm{R}$, gttgaaggtagtttcgtgga. A miScript reverse transcription kit was used to reverse transcribe RNA into cDNA and a miScript SYBR-Green PCR kit (both Qiagen Nordic) was used for qPCR to detect the expression of miR-199a-5p. The specific primers were as follows: miRNA-199a: F, tcccagtgt tcagactacc; R, tttggcactagcacatt; U6: F, ctcgcttcggcagcaca; $\mathrm{R}$, aacgcttcacgaatttgcgt. The expression of U6 was used as an endogenous control. Data were analyzed using the $2^{-\Delta \Delta C T}$ method.

Western blot analysis. Total protein $(60 \mu \mathrm{g})$ extracted from the indicated cells was separated on SDS-polyacrylamide gels for E-cadherin and GAPDH detection. GAPDH was used as a loading control. The protein in gels was transferred to nitrocellulose membranes, and then incubated with the indicated antibodies at the recommended dilutions overnight at $4^{\circ} \mathrm{C}$. Next, the membranes were washed with $0.1 \mathrm{M}$ phosphate-buffered saline with Tween-20 and incubated with horseradish peroxidase-conjugated secondary antibody. Signals were visualized using enhanced chemiluminescence substrates and quantified using Optiquant 3.0 software (PerkinElmer, Inc., Waltham, MA, USA).
Invasion assay. Cells were cultivated to $80 \%$ confluence on 12 -well plates. Then, cellular growth procedures were observed at $24 \mathrm{~h}$. All the experiments were repeated in triplicate. Transwell invasion chambers were used to evaluate cell invasion. Then cells invading across the membrane were counted under a light microscope (YS100; Nikon Corporation, Tokyo, Japan).

Gelatin zymography. An MMP zymography assay kit (Applygen Technologies, Inc., Beijing, China) was used to assess the activity of MMP2 and MMP9. Protein extracts and positive mixture were mixed with an equal volume of $2 \mathrm{X}$ sodium dodecyl sulphate (SDS)-polyacrylamide gel electrophoresis non-reducing buffer, and electrophoresed on $8 \%$ SDS polyacrylamide gels containing $2 \mathrm{mg} / \mathrm{ml}$ gelatin. Gels were then washed twice for $30 \mathrm{~min}$ in buffer A at room temperature, and incubated for $4 \mathrm{~h}$ at room temperature in incubation buffer B. Gels were then stained for $2 \mathrm{~h}$ with $0.25 \%$ Coomassie brilliant blue and then destained in destaining buffer (10\% acetic acid and 20\% methanol) for $60 \mathrm{~min}$.

Statistical analysis. Data were expressed as the means \pm standard deviation and analyzed by Student's t-test. $\mathrm{P}<0.05$ compared with respective controls was considered to indicate a statistically significant difference.

\section{Results}

miR-199a-5p regulates the expression levels of E-cadherin in cSCC. E-cadherin is downregulated in cSCC tumors (19). To investigate whether the downregulation of E-cadherin is regulated by miR-199a-5p, miR-199a-5p mimics and inhibitors were transfected into A-431 cells and E-cadherin expression was assessed by qPCR and western blot analysis. qPCR was used to measure the expression of miR-199a-5p following treatment with miR-199a-5p mimics or inhibitor. The qPCR results revealed that miR-199a-5p mimics efficiently induced the expression of miR-199a, and miR-199a-5p inhibitor significantly downregulated the expression of miR-199a (Fig. 1A). 

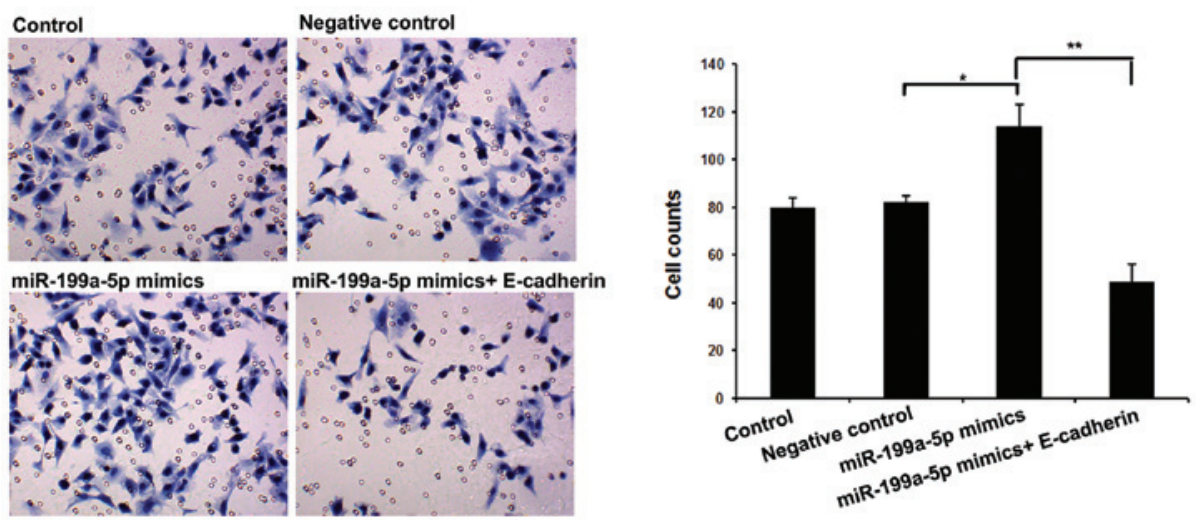

Figure 2. Transwell analysis revealed the effect of miR-199a-5p on cutaneous squamous cell carcinoma cell invasion. A-431 cells were transfected with miR-199a-5p mimics alone or in combination with E-cadherin. Error bars represent the means \pm standard error. "P<0.01 vs. negative control group; ${ }^{* *} \mathrm{P}<0.01$ vs. miR-199a-5p mimics group.

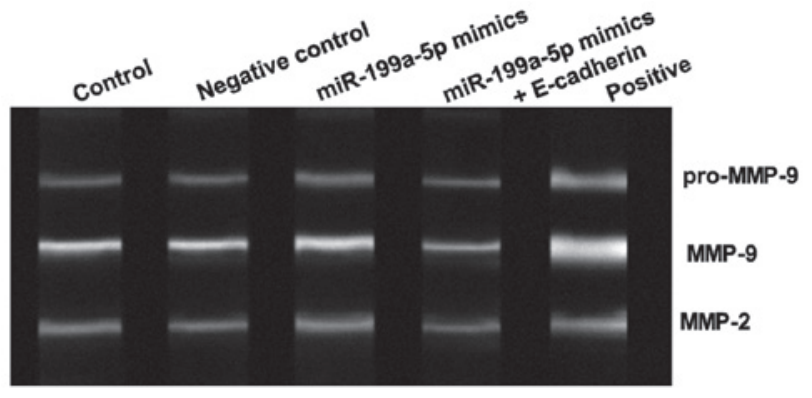

Figure 3. Effect of miR-199a-5p on the activation of matrix metalloproteinase (MMP)2 and MMP 9. Activation of MMP2/9 was evaluated by gelatin zymography. A-431 cells were transfected with miR-199a-5p alone or in combination with E-cadherin.

qPCR and western blot analysis revealed that ectopic expression of miR-199a-5p by transfection of miR-199a-5p mimic led to decreased expression of E-cadherin, and that knockdown of miR-199a-5p by miR-199a-5p inhibitors upregulated the expression of E-cadherin (Fig. 1B and C). Additionally, E-cadherin and miR-199a-5p mimics were cotransfected into A-431 cells. miR-199a-5p expression was not affected by E-cadherin transfection. However, the miRNA and protein expression of E-cadherin were greatly increased in the miR-199a-5p mimics + E-cadherin group (A-431 cells cotransfected with E-cadherin and miR-199a-5p mimics) compared with that in the miR-199a-5p mimics group. Together, the above results confirmed that E-cadherin is one of the downstream genes of miR-199a-5p in cSCC cells, and that overexpression of miR-199a-5p induces the repression and downregulates the expression of E-cadherin.

Overexpression of miR-199a-5p represses $c S C C$ cell invasion To further explore the function of miR-199a-5p in cSCC cells, we evaluated cell invasion by Transwell following transfection with miR-199a-5p mimics alone or combined with E-cadherin. As shown in Fig. 2, we observed that overexpression of miR-199a-5p significantly increased cell invasion, which was reversed by cotransfection with miR-199a-5p mimics and E-cadherin. Together, the above results may indicate that miR-199-5p performs an oncogenic role by suppressing E-cadherin expression in cSCC.
Overexpression of miR-199a-5p affects activity of MMP2 and MMP9 in $C S C C$. In addition, we assessed the effects of miR-199a-5p on the activity of MMP2 and MMP9. As shown in Fig. 3, the activity of MMP2 and MMP9 was significantly upregulated by miR-199a-5p mimics compared with the scramble control, and this was reversed by cotransfection with miR-199a-5p mimics and E-cadherin. This suggested that upregulation of miR-199a-5p increased the activity of MMP2 and MMP9 by regulating E-cadherin expression in cSCC cells.

\section{Discussion}

Overall, the morbidity of cSCC has been increasing worldwide over the past 10 years. The 5-year overall survival rate of patients with metastatic cSCC is $\sim 50 \%$ lower than that of patients with regional or localized disease (20). A better understanding of the genes or molecular alterations involved in cSCC progression is likely to be useful in early detection and future targeted treatment strategies. Previously, multiple studies have revealed that miRNA expression is a crucial regulator of cSCC progression $(9,21,22)$. In this study, we demonstrated that miR-199a-5p was an upstream regulator of E-cadherin, and that it suppressed the expression of E-cadherin in cSCC cells. In addition, miR-199a-5p significantly decreased cell invasion and the activity of MMP2 and MMP9 in cSCC cells.

E-cadherin is a $120 \mathrm{kDa}$ calcium-dependent transmembrane glycoprotein encoded by the $\mathrm{CDH} 1$ gene. It is expressed in the majority of epithelial cells. E-cadherin, as a crucial protein-mediated cell-cell adhesion molecule, plays an essential role in establishing cell polarity and in maintaining normal tissue architecture. Loss of E-cadherin decreases cellular adhesion, resulting in an increase in cellular motility (23). Accumulating studies have demonstrated that E-cadherin is closely associated with tumor invasion and metastasis (24). E-cadherin is involved in the process of epithelial-to-mesenchymal transition, which contributes to intercellular adhesion, acquisition of a mesenchymal phenotype and enhanced migratory potential in tumors. It has been reported that downregulation of E-cadherin in esophageal carcinoma was associated with an increased invasive and metastatic potential (25). A previous study has revealed that 
E-cadherin, as an epithelial marker, is downregulated in cSCC (26). As lower expression of E-cadherin in cSCC tumors and cells has been confirmed in previous studies $(19,27)$, the direct upstream miRNAs of E-cadherin have become increasing significant in understanding the mechanism of E-cadherin-involved tumor progression. In a previous study, the downregulated expression of miRNA-199a was observed in liver, breast and bladder cancer (28). It was reported that inducing the expression of miRNA-199a was responsible for decreasing the proliferation of gastric cancer cells by targeting the mTOR signaling pathway (29). The abundant expression of miR-199a-5p has been observed in liver tissue, and in epithelial as well as non-epithelial tissues (30). Inhibition of miR-199a-5p impaired the metastatic potential of gastric cancer cells, and E-cadherin was identified as a direct and functional target of miR-199a-5p in gastric cancer cells (31). In the present study, we confirmed that E-cadherin was one of the downstream genes of miR-199a-5p in cSCC cells, and that overexpression of miR-199a-5p induced the repression and downregulated the expression of E-cadherin in $\mathrm{CSCC}$.

MMPs are considered to play an essential role in the metastasis of tumor cells by decomposing the extracellular matrix and destroying the basement membrane of blood vessels. The dissolution of the basement membrane caused by MMP in tumors is a notable process in the movement of tumor cells. A significant correlation has been reported between the activation of MMP and the migration and invasion of tumor cells $(32,33)$. The expression of MMP2 and MMP9 was observed to be higher in patients with lymph node metastases compared with patients without lymph node involvement in oropharyngeal SCC (34). By gelatin zymography, we observed that transfection of miR-199a-5p mimic induced the activity of MMP2 and MMP9. Inversely, the activity of MMP2 and MMP9 was reduced by cotransfection with miR-199a-5p mimic and E-cadherin. Thus, the oncogenic role of miR-199a-5p in cell invasion in cSCC cells may be associated with the activity of MMP2 and MMP9.

In conclusion, we demonstrated that miR-199a-5p specifically suppressed the expression of E-cadherin at the mRNA and protein level in cSCC cells. Moreover, the oncogenic role of miR-199a in cell invasion in cSCC cells was also associated with the activity of MMP2 and MMP9. This miR-199a-5p/E-cadherin/MMP pathway may therefore offer a novel perspective for cSCC metastasis and may represent an effective therapeutic target for cSCC treatment.

\section{References}

1. Alam M and Ratner D: Cutaneous squamous-cell carcinoma. N Engl J Med 344: 975-983, 2001.

2. Ratushny V, Gober MD, Hick R, Ridky TW and Seykora JT: From keratinocyte to cancer: the pathogenesis and modeling of cutaneous squamous cell carcinoma. J Clin Invest 122: 464-472, 2012.

3. Bushati N and Cohen SM: MicroRNA functions. Annu Rev Cell Dev Biol 23: 175-205, 2007.

4. Huang S and He X: The role of microRNAs in liver cancer progression. Br J Cancer 104: 235-240, 2011.

5. Kent OA and Mendell JT: A small piece in the cancer puzzle: microRNAs as tumor suppressors and oncogenes. Oncogene 25: 6188-6196, 2006.

6. Acunzo M, Romano G, Wernicke D and Croce CM: MicroRNA and cancer - A brief overview. Adv Biol Regul 57: 1-9, 2015.
7. Lee JW, Choi CH, Choi JJ, Park YA, Kim SJ, Hwang SY, Kim WY, Kim TJ, Lee JH, Kim BG and Bae DS: Altered MicroRNA expression in cervical carcinomas. Clin Cancer Res 14: 2535-2542, 2008.

8. Bruegger C, Kempf W, Spoerri I, Arnold AW, Itin PH and Burger B: MicroRNA expression differs in cutaneous squamous cell carcinomas and healthy skin of immunocompetent individuals. Exp Dermatol 22: 426-428, 2013.

9. Sand M, Skrygan M, Georgas D, Sand D, Hahn SA, Gambichler T, Altmeyer P and Bechara FG: Microarray analysis of microRNA expression in cutaneous squamous cell carcinoma. J Dermatol Sci 68: 119-126, 2012.

10. Zhou M, Liu W, Ma S, Cao H, Peng X, Guo L, Zhou X, Zheng L, Guo L, Wan M, et al: A novel onco-miR-365 induces cutaneous squamous cell carcinoma. Carcinogenesis 34 : 1653-1659, 2013.

11. Wang A, Landén NX, Meisgen F, Lohcharoenkal W, Ståhle M, Sonkoly E and Pivarcsi A: MicroRNA-31 is overexpressed in cutaneous squamous cell carcinoma and regulates cell motility and colony formation ability of tumor cells. PLoS One 9: e103206, 2014.

12. Gastaldi C, Bertero T, Xu N, Bourget-Ponzio I, Lebrigand K, Fourre S, Popa A, Cardot-Leccia N, Meneguzzi G, Sonkoly E, et al: MiR-193b/365a cluster controls progression of epidermal squamous cell carcinoma. Carcinogenesis 35: 1110-1120, 2014.

13. Yanaihara N, Caplen N, Bowman E, Seike M, Kumamoto K, Yi M, Stephens RM, Okamoto A, Yokota J, Tanaka T, et al: Unique microRNA molecular profiles in lung cancer diagnosis and prognosis. Cancer cell 9: 189-198, 2006.

14. Huang L, Lin JX, Yu YH, Zhang MY, Wang HY and Zheng M: Downregulation of six microRNAs is associated with advanced stage, lymph node metastasis and poor prognosis in small cell carcinoma of the cervix. PLoS One 7: e33762, 2012.

15. Shen Q, Cicinnati VR, Zhang X, Iacob S, Weber F, Sotiropoulos GC, Radtke A, Lu M, Paul A, Gerken G and Beckebaum S: Role of microRNA-199a-5p and discoidin domain receptor 1 in human hepatocellular carcinoma invasion. Mol Cancer 9: 227, 2010.

16. Xu N, Zhang J, Shen C, Luo Y, Xia L, Xue F and Xia Q: Cisplatin-induced downregulation of miR-199a-5p increases drug resistance by activating autophagy in HCC cell. Biochem Biophys Res Commun 423: 826-831, 2012.

17. He XJ, Ma YY, Yu S, Jiang XT, Lu YD, Tao L, Wang HP, Hu ZM and Tao HQ: Up-regulated miR-199a-5p in gastric cancer functions as an oncogene and targets klotho. BMC cancer 14: 218,2014

18. Hu Y, Liu J, Jiang B, Chen J, Fu Z, Bai F, Jiang J and Tang Z: MiR-199a-5p loss up-regulated DDR1 aggravated colorectal cancer by activating epithelial-to-mesenchymal transition related signaling. Dig Dis Sci 59: 2163-2172, 2014.

19. Barrette K, Van Kelst S, Wouters J, Marasigan V, Fieuws S, Agostinis P, van den Oord J and Garmyn M: Epithelial-mesenchymal transition during invasion of cutaneous squamous cell carcinoma is paralleled by AKT activation. Br J Dermatol 171: 1014-1021, 2014.

20. Balch CM, Gershenwald JE, Soong SJ, Thompson JF, Atkins MB, Byrd DR, Buzaid AC, Cochran AJ, Coit DG, Ding S, et al: Final version of 2009 AJCC melanoma staging and classification. J Clin Oncol 27: 6199-6206, 2009.

21. Tanemura A, Terando AM, Sim MS, van Hoesel AQ, de Maat MF, Morton DL and Hoon DS: CpG island methylator phenotype predicts progression of malignant melanoma. Clin Cancer Res 15: 1801-1807, 2009.

22. Xu N, Zhang L, Meisgen F, Harada M, Heilborn J, Homey B, Grandér D, Ståhle M, Sonkoly E and Pivarcsi A: MicroRNA-125b down-regulates matrix metallopeptidase 13 and inhibits cutaneous squamous cell carcinoma cell proliferation, migration and invasion. J Biol Chem 287: 29899-29908, 2012.

23. Nelson WJ and Nusse R: Convergence of Wnt, beta-catenin and cadherin pathways. Science 303: 1483-1487, 2004.

24. Deng QW, He BS, Pan YQ, Sun HL, Xu YQ, Gao TY, Li R, Song GQ and Wang SK: Roles of E-cadherin (CDH1) genetic variations in cancer risk: a meta-analysis. Asian Pac J Cancer Prev 15: 3705-3713, 2014.

25. Shimada Y, Hashimoto Y, Kan T, Kawamura J, Okumura T, Soma T, Kondo K, Teratani N, Watanabe G, Ino Y, et al: Prognostic significance of dysadherin expression in esophageal squamous cell carcinoma. Oncology 67: 73-80, 2004. 
26. Toll A, Masferrer E, Hernández-Ruiz ME, Ferrandiz-Pulido $\mathrm{C}$, Yébenes M, Jaka A, Tuneu A, Jucglà A, Gimeno J, Baró T, et al: Epithelial to mesenchymal transition markers are associated with an increased metastatic risk in primary cutaneous squamous cell carcinomas but are attenuated in lymph node metastases. J Dermatol Sci 72: 93-102, 2013.

27. Brouxhon SM, Kyrkanides S, Raja V, Silberfeld A, Teng X, Trochesset D, Cohen J and Ma L: Ectodomain-specific E-cadherin antibody suppresses skin SCC growth and reduces tumor grade: a multitargeted therapy modulating RTKs and the PTEN-p53-MDM2 axis. Mol Cancer Ther 13: 1791-1802, 2014.

28. Jiang J, Gusev Y, Aderca I, Mettler TA, Nagorney DM, Brackett DJ, Roberts LR and Schmittgen TD: Association of MicroRNA expression in hepatocellular carcinomas with hepatitis infection, cirrhosis and patient survival. Clin Cancer Res 14: 419-427, 2008

29. Peng W, Chen ZY, Wang L, Wang Z and Li J: MicroRNA-199a-3p is downregulated in gastric carcinomas and modulates cell proliferation. Genet Mol Res 12: 3038-3047, 2013.

30. Liang Y, Ridzon D, Wong L and Chen C: Characterization of microRNA expression profiles in normal human tissues. BMC Genomics 8: 166, 2007.
31. Zhao X, He L, Li T, Lu Y, Miao Y, Liang S, Guo H, Bai M, Xie H, Luo G, et al: SRF expedites metastasis and modulates the epithelial to mesenchymal transition by regulating miR-199a-5p expression in human gastric cancer. Cell Death Differ 21: 1900-1913, 2014

32. Ryzhakova OS and Solov'eva NI: Matrix metalloproteinases (MMP)-MMP-1,-2,-9 and its endogenous activity regulators in transformed by E7 oncogene HPV16 and HPV18 cervical carcinoma cell lines. Biomed Khim 59: 530-540, 2013 (In Russian).

33. Mohtasham N, Babakoohi S, Shiva A, Shadman A, Kamyab-Hesari K, Shakeri MT and Sharifi-Sistani N: Immunohistochemical study of p53, Ki-67, MMP-2 and MMP-9 expression at invasive front of squamous cell and verrucous carcinoma in oral cavity. Pathol Res Pract 209: 110-114, 2013.

34. Burduk PK, Bodnar M, Sawicki P, Szylberg Ł, Wiśniewska E, Kaźmierczak W, Martyńska M and Marszałek A: Expression of metalloproteinases 2 and 9 and tissue inhibitors 1 and 2 as predictors of lymph node metastases in oropharyngeal squamous cell carcinoma. Head Neck 37: 418-422, 2015. 